\title{
LEIAUTE DE SALAS DE COORDENAÇÃO BIM: CRITÉRIOS E MÉTRICAS DE AVALIAÇÃO'
}

\section{BIM COORDINATION ROOM LAYOUT: CRITERIA AND METRICS FOR ASSESSMENT}

\author{
Miriam Roux Azevedo Addor \\ Universidade de São Paulo, USP \\ miriamaddor@usp.br \\ Eduardo Toledo Santos \\ Universidade de São Paulo, USP \\ etoledo@usp.br
}

\begin{abstract}
Resumo
Ao se coordenar projetos em BIM, são requeridas mídias diferentes daquelas utilizadas quando a análise é baseada em papel. Surgem novas necessidades aos participantes da reunião como visualizar, navegar ou verificar um modelo 3D, fazer croquis digitais, e outras atividades que devem tornar a reunião presencial produtiva, com o objetivo de resolver os problemas dos projetos. O objetivo deste artigo é apresentar critérios e métricas para avaliação da qualidade de leiautes de salas de coordenação em BIM (BIM Rooms). Para validar os resultados, foram analisados e avaliados 14 leiautes diferentes para uma mesma sala, em relação às métricas e critérios relacionados a esse aspecto. Com o propósito de estabelecer qual a relação de importância entre todos os 10 critérios propostos para atendimento as necessidades dos participantes de reuniões BIM foi aplicado o método AHP de decisão multicritério baseado na análise de comparações em pares. Os resultados permitiram determinar os melhores leiautes para uma sala equipada com 12 participantes e 2 projetores de curta distância. Os critérios e métricas apresentados, bem como a metodologia para medi-los, são úteis para a avaliação de outras salas e leiautes para atender aos usuários de salas de reuniões de projetos baseados em BIM.
\end{abstract}

Palavras-chave: Coordenação de Projetos. Modelagem da Informação da Construção. Métrica. AHP.

\begin{abstract}
Coordination of BIM-based projects requires distinct media from those used for paper-based analysis. Participants of BIM coordination meetings have new needs as visualizing, navigating or checking 3D models, preparing digital sketches and other tasks that make a face-to-face meeting effective for solving design problems. The aim of this paper is to present criteria and metrics for assessing the quality of BIM Room layouts. Ten criteria were defined regarding the six main user needs as well as 12 metrics to provide scores for valuing those criteria. For validating our results, 14 different layouts for the same room were analyzed and assessed using the criteria and metrics related to layout. The AHP - Analytic Hierarchy Process method for multi-criteria decision making based on pairwise comparisons was used for determining the relative importance among the established criteria for fulfilling meeting participant's needs. The results allowed determining the best layouts for rooms equipped with 6 desks (12 people) and 2 short-throw projectors. The proposed criteria and metrics, as well as their measuring methodology, are useful for the assessment of other rooms and layouts, supporting the design of better facilities for fulfilling the needs of participants in BIM coordination meetings.
\end{abstract}

Keywords: Project Coordination. Building Information Modeling. Metric. AHP.

\footnotetext{
1 ADDOR, M. R. A.; SANTOS, E. T. Leiaute de salas de coordenação BIM: critérios e métricas de avaliação. In: ENCONTRO BRASILEIRO DE TECNOLOGIA DE INFORMAÇÃO E COMUNICAÇÃO NA CONSTRUÇÃO, 7., 2015, Recife. Anais... Porto Alegre: ANTAC, 2015.
} 


\section{2}

\section{INTRODUÇÃO}

Um estudo publicado sobre o valor dos negócios em construção em BIM - Modelagem da Informação da Construção nos maiores mercados globais mostra que, no Brasil, $40 \%$ das empresas do setor de projeto e construção que responderam à pesquisa se encontram em nível médio de implantação de BIM, sendo que 70\% destas empresas vêm usando BIM de 1 a 2 anos (MCGRAW-HILL CONSTRUCTION, 2014). Este é um indicador da realidade brasileira de avanço neste novo processo de trabalho. BIM é um processo de produção, uso e atualização de um modelo de informações da construção durante todo o seu ciclo de vida. Esse modelo, além da geometria da obra, contém numerosas informações sobre seus diferentes aspectos, podendo abranger todas as disciplinas envolvidas em um empreendimento (SANTOS, 2012). Modelos BIM devem ser interpretados e analisados nas diversas fases do ciclo de vida seja no projeto, construção ou pós-obra. Para isso pressupõe-se colaboração (LEICHT,2009). Colaboração em projetos é definida como um processo onde os projetistas se comunicam de forma dinâmica e trabalham em conjunto, com o objetivo de estabelecer coletivamente os objetivos do projeto e construir as soluções. A colaboração implica no trabalho em equipe, negociação e representações compartilhadas. (LAHTI et al., 2004). De acordo com este autor, o processo de projeto colaborativo apresenta três padrões característicos e de intensidade variável: a coordenação, a cooperação e a colaboração. O enfoque deste artigo restringe-se à fase de coordenação de projetos e à infraestrutura e instalações físicas necessárias para o funcionamento das atividades de coordenação e interpretação de modelos BIM em reuniões presenciais dentro de salas de coordenação.

A coordenação de projetos é uma atividade multidisciplinar de suporte ao processo de projeto focada no gerenciamento de questões técnicas e tomada de decisão no projeto (MELHADO et al., 2005). Esta atividade geralmente implica na comunicação entre todos os projetistas, tanto dentro quanto fora das salas de reunião de projetos. Os espaços onde as reuniões de coordenação de projetos ocorrem podem ser físicos ou virtuais (LISTON et al., 2000) e são utilizados para compartilhamento de informações e para consultas e tomada de decisão sobre as questões dos projetos. A tomada de decisão em projeto, na maioria das vezes, ainda é feita presencialmente (LISTON et al., 2000).

A adoção do processo BIM na fase de projeto e a disponibilidade de novas tecnologias estão levando os usuários a buscar alternativas para a infraestrutura do espaço onde estas reuniões são realizadas (LISTON et al. 2000; FRUCHTER et al. 2006; DENNIS et al, 2008; LEICHT, 2009), apesar da crescente disponibilidade de infraestrutura de internet possibilitar às equipes execução de reuniões a distância.

O foco deste trabalho está direcionado para o estudo de reuniões face a face, procurando enfatizar a resolução dos problemas, decisões de projeto e a diminuição do período de latência entre os participantes das reuniões. Nos últimos vinte anos, o formato dos espaços de reunião vem se modificando. Esta alteração pode ser explicada tanto pelo acesso a novas tecnologias como pela forma de projetar, passando das formas tradicionais de projeto em 2D para formas tridimensionais paramétricas orientadas ao objeto.

Com o objetivo de identificar as atividades que ocorrem com maior frequência dentro das salas de coordenação de projetos, foi realizado um estudo em vinte e seis reuniões de coordenação baseadas em análise de projetos em 2D (ADDOR; SANTOS, 2014). Identificou-se que $48 \%$ das ações que ocorrem dentro das salas de coordenação dizem respeito a visualizar plantas impressas por alguns ou todos os participantes da reunião.

Embora este estudo tenha sido feito sobre reuniões baseadas em análise sobre papel, de acordo com a quantidade de ações ocorridas, chegou-se à conclusão que uma grande tela de projeção poderia suprir a maioria das ações mais frequentes nestas reuniões. Foram 
também observadas reuniões em processo BIM e como resultado foram extraídas várias necessidades dos usuários em função da mudança de padrão de comunicação e comportamento dos usuários frente à tecnologia e que serviram de base para estruturação da metodologia deste trabalho. Com base na abordagem de Pesquisa Construtiva (Constructive Research), foi determinado o problema principal da pesquisa (OYEGOKE, 2011; KASANEN et al., 1993): identificar qual a infraestrutura mais otimizada para uma sala de coordenação em BIM.

O objetivo deste artigo é apresentar uma metodologia para determinação de leiaute otimizado para salas de coordenação em BIM, enfocando a captação das necessidades dos usuários, criação de métricas para avaliação e análise de decisão multicritério e apresentar os resultados obtidos num estudo de caso.

\section{AVALIAÇÃO DAS NECESSIDADES DOS USUÁRIOS DE UMA SALA DE COORDENAÇÃO BIM}

Antes de planejar o uso de um espaço de trabalho interativo, primeiro é necessário entender quais são os requisitos que definem os tipos de interação relevantes. Essas interações podem ser de caráter informacional ou físico. Algumas características foram identificadas por Rankin et al. (2007) e dizem respeito a:

- Visualização de documentos em grandes telas;

- Revisar documentos e interagir com a informação na sala de reunião;

- Permitir comunicação mais eficaz entre os participantes, diminuindo a perda ou má interpretação de informações;

- Dar suporte a documentação eletrônica e aprendizagem colaborativa e permitir que a equipe traga menos informações em papel para as reuniões;

- Promover a interação pessoal das equipes de trabalho;

- Acessar as informações do projeto em um ambiente mais descontraído e relaxado;

- Fazer uso de servidor na sala e de rede de computadores;

- Fazer uso de telas táteis;

- Prover equipamentos permanentes e portáteis na sala. Os permanentes incluem telas touch screen, servidor, teclados sem fio, mouse sem fio e apontadores a laser. Os portáteis incluem notebooks pessoais, tablets, smartphones;

- Fazer gravações em vídeo e áudio.

Pesquisas anteriores na área focaram na observação de participantes de reuniões com uma variedade de equipamentos que proporcionaram 0 acesso aos dados, simulações, visualização e trocas entre os participantes presenciais, em vez de se preocuparem em antecipar as necessidades dos usuários ou tornar o espaço "inteligente" (JOHANSON et al., 2002; GOLDPARVAR-FARD et al., 2006). Baseado neste tipo de conceituação, para o presente estudo foram feitas e analisadas dez gravações em vídeo de reuniões de coordenação de projetos em BIM. O objetivo da análise destas gravações foi o de identificar o maior número de necessidades dos usuários em relação à infraestrutura física, aos equipamentos, mobiliário e inter-relacionamento entre os participantes. Além da análise das gravações em vídeo foram consultadas normas técnicas vigentes relativas à ergonomia da relação humano-sistema (ABNT NBR ISO 9241-210:2011; ABNT ISO 9241-11:2011) onde a abordagem da experiência do usuário é consequência da apresentação, funcionalidade, desempenho do sistema, comportamento da interação e capacidades assistivas de um sistema interativo, tanto em hardware quanto em software. A primeira norma preconiza a necessidade de se definir o contexto de uso atual dos sistemas, as características dos usuários, das tarefas e dos ambientes organizacionais, técnicos e físicos. Estes aspectos foram considerados importantes como requisitos para levantamento de dados dos usuários de salas de reunião, tais como: 
- Identificar quais são os usuários da sala;

- Identificar quais são as características destes usuários (conhecimentos, habilidades, experiência, formação, treinamento, aptidões);

- Identificar os objetivos e tarefas dos usuários dentro da sala;

- Identificar as características relevantes do ambiente físico, social e cultural dos usuários;

- Especificar os requisitos dos usuários (necessidades dos usuários, contexto de uso, ergonomia, interação, mobiliário, infraestrutura, espaço, conforto ambiental).

A metodologia para captura das necessidades dos usuários na sala de coordenação BIM levou em conta:

- Mapeamento de observações de reuniões de coordenação em BIM;

- Normas técnicas vigentes;

- Experiência da autora em mais de 25 anos de coordenação de projetos.

\section{DEFINIÇÃO DOS REQUISITOS PARA ANÁLISE DE LEIAUTE DE UMA SALA DE COORDENAÇÃO BIM}

Alguns achados do estudo elaborado sobre padrões de comunicação e requisitos em salas de coordenação de projetos (ADDOR; SANTOS, 2014) forneceram a base para definição dos requisitos para a análise dos leiautes. Apesar de esta análise ter sido feita sobre projetos tradicionais (não BIM), é importante ressaltar que somente ações que necessitem de suporte de infraestrutura foram consideradas e que tomar decisões, resolver questões de projeto e garantir a melhoria do padrão do projeto são objetivos principais a serem atendidos, tanto em reuniões baseadas em análise sobre papel, como em reuniões em BIM. Além desta base de dados, também foram considerados os dados de observação de 10 gravações em vídeo de reuniões de coordenação baseadas em BIM, conforme prescreve a norma ISO 9241-2:1992 no estudo observacional dos usuários para estabelecer requisitos para as tarefas.

As necessidades dos usuários a seguir foram selecionadas para compor os critérios e métricas a serem aplicados para avaliação dos leiautes de salas de coordenação BIM:

- Visualização de informações: necessidade de todos conseguirem visualizar um projeto, uma imagem, um texto, uma planilha de dados ou um vídeo;

- Interatividade: necessidade de comunicação e interação interpessoal de modo que os participantes possam enxergar uns aos outros e se comunicar;

- Infraestrutura elétrica/rede: necessidade que a sala deve atender para ligar e conectar equipamentos de usuários externos, tais como notebook, tablets ou smartphones;

- Mobiliário: necessidade que a sala deve satisfazer a fim de proporcionar conforto e assento aos usuários e apoio para equipamentos externos e objetos pessoais, tais como notebook, caderno de anotações, tablets;

- Espaço Físico: necessidade que a sala deve satisfazer com relação à circulação dos usuários, colocação do mobiliário e espaço para assento das pessoas;

- Conforto Ambiental: parâmetros de iluminação, temperatura e acústica mínimos necessários para que os usuários possam participar com conforto de uma reunião.

\section{METODOLOGIA}

O método de pesquisa adotado foi a realização de levantamento bibliográfico relativo às questões dos critérios dos leiautes apresentadas acima, com o apoio de normas técnicas e padrões estabelecidos de ergonomia. Com base neste levantamento, foram definidos critérios e métricas para as principais necessidades dos usuários. Estas métricas foram aplicadas em quatorze versões de leiaute para uma mesma sala disponível para o estudo. 
Estes leiautes continham a mesma quantidade de mesas, cadeiras e telas de projeção e acomodavam o mesmo número de usuários. As métricas foram aplicadas e determinadas as notas para cada leiaute. Para que estas variáveis pudessem ser comparadas equilibradamente, foi aplicado o método AHP - Analytic Hierarchy Process (SAATY, 1990). Este método seleciona os fatores a serem analisados, classifica-os em uma organização hierárquica e os compara par a par. Esta comparação leva em conta a importância de um fator sobre o outro para o critério analisado. Os resultados obtidos são medidas relativas a partir das comparações.

Por fim os resultados foram analisados e chegou-se a conclusão sobre quais leiautes são os mais indicados para uso da sala estudada para reuniões de coordenação BIM, levando-se em consideração os critérios visualização, interatividade e espaço físico. Os critérios de infraestrutura elétrica/rede, mobiliário e conforto ambiental não foram considerados na análise, pois são sempre os mesmos já que apenas uma única sala física foi considerada no estudo.

\subsection{Criação de Leiautes}

Para este estudo foram concebidos 14 leiautes contendo 6 mesas de $1,20 \mathrm{~m}$ (comprimento) x 0,60m (largura) x 0,77m (altura), com 12 cadeiras, 12 participantes, dois projetores interativos com telas móveis de 96 polegadas, contidos em uma sala de reuniões de $5,96 \mathrm{~m}$ $X 4,71 \mathrm{~m}$. Considerando estes elementos, os leiautes analisados apresentam-se conforme figura 1 abaixo:

Figura 1- Leiautes

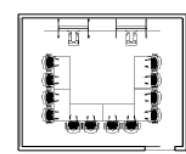

L1

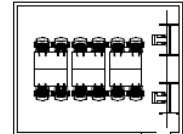

L5

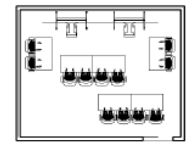

L9

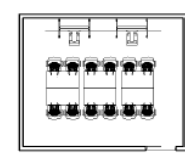

L2

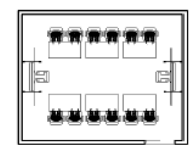

L6

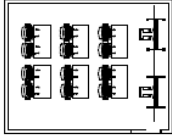

L10

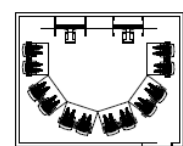

L13

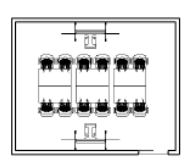

L3

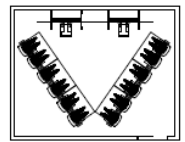

L7

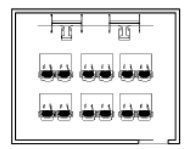

L11

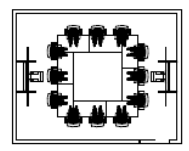

L14

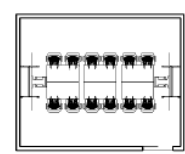

L4

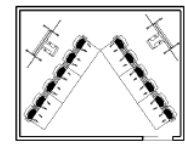

L8

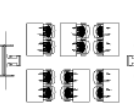

L12

Fonte: Autores

\subsection{Determinação de critérios e métricas}

Para cada uma das 6 necessidades dos usuários levantadas no item 3 deste artigo, foram criados critérios e métricas para avaliação dos leiautes, descritos abaixo: 
Visualização de Informações: Para esta necessidade foram estabelecidos 3 critérios que dizem respeito ao relacionamento entre o participante da reunião e a tela de projeção:

Critério 1: para visualização confortável da tela, o ângulo horizontal (A) com que o observador tem que girar o olho, a cabeça e/ou o corpo para enxergar toda a área de visualização deve ser baixo. Para que um observador não precise mexer o olho ao ver algum objeto ou imagem a variação de ângulo é de 0 a 20 graus para cada lado. Se esta variação estiver entre 20 e 35 graus o observador terá que mexer o olho. Se for entre 35 e 55 graus, terá que mexer a cabeça e, se maior que 55 graus, terá que girar o corpo ou a cadeira em que estiver sentado (PANERO; ZELNIK, 1979). Tomando como base estes critérios, foi estabelecida uma métrica que mede o ângulo entre as arestas extrema esquerda e direita da tela, tendo o observador no vértice. Os ângulos foram categorizados em 4 faixas: "menor que 40 graus"; "entre 40 e 70 graus"; "entre 70 e 110 graus" e "acima de 110 graus". Estes ângulos foram classificados em faixas de acordo com a análise AHP e calculou-se a média das classificações.

Critério 2: a porcentagem de oclusão da tela percebida pelo observador a partir de seu posicionamento na sala, considerando os demais participantes, deve ser pequena. Para avaliar sob este critério, a métrica adotada é a porcentagem da tela bloqueada pelo corpo dos demais participantes (supondo um manequim padronizado) a partir do ponto de vista do participante considerado. Para fazer essa medição, uma imagem do ambiente a partir desta posição é gerada em aplicativo de modelagem 3D e exportada para aplicativo de tratamento e análise de imagem onde são contadas as quantidades de pixels ocultos e não ocultos na região da tela (ferramenta de histograma). Da relação entre as duas áreas é calculada a área de oclusão da tela. Ao invés de modelo virtual, é possível a utilização de fotografia da sala com participantes típicos, porém este processo é mais demorado e trabalhoso. Classificou-se o resultado das oclusões de acordo com as faixas obtidas na análise AHP e calculou-se a média desta classificação.

Critério 3: o máximo ângulo vertical entre a aresta superior da tela e a horizontal na altura do observador deve ser pequeno. Para cálculo da métrica correspondente, numa vista em corte, traçar uma perpendicular do usuário até o centro da tela de projeção. Medir o ângulo $\alpha$ entre o usuário e a aresta superior da tela. Os ângulos foram categorizados em faixas: $0^{\circ}<\alpha \leq 30^{\circ} ; 30^{\circ}<\alpha \leq 50^{\circ}$; e $\alpha>50^{\circ}$ (PANERO, ZELNIK; 1979). Classificou-se os ângulos de acordo com as faixas obtidas na análise AHP e calculou-se a média desta classificação.

Interatividade: Para esta necessidade foi estabelecido um critério que medisse o grau de relacionamento e interatividade entre os participantes da reunião. Para que este critério fosse composto, foram definidas 3 métricas: obstrução do participante (zero se locutor consegue enxergar ou 1 se locutor não enxerga o interlocutor diretamente); distância (entre o locutor e o interlocutor) e ângulo que o locutor tem que girar para interagir com o interlocutor. Para distância foram consideradas 4 faixas: até 1,40m; de 1,40 a 2,40m; de 2,40 a 3,00m e; acima de $3,00 \mathrm{~m}$. Para o ângulo foram consideradas 4 faixas: $0^{\circ}$ a $45^{\circ} ; 45^{\circ}$ a $90^{\circ} ; 90^{\circ}$ a $135^{\circ}$ e; $135^{\circ}$ a $180^{\circ}$. A referência para estas métricas foi obtida através de estudo observacional em reuniões de coordenação de projeto. Para a métrica da obstrução, o posicionamento de cada participante no leiaute foi analisado: se não houvesse obstrução foi categorizado com 0 e se houvesse com 1. Classificou-se cada uma destas métricas de acordo com a faixa obtida na análise AHP e calculou-se a média desta classificação.

Infraestrutura: O critério estabelecido para esta necessidade diz respeito à quantidade de tomadas de uso geral que há na sala para ligar os equipamentos dos participantes da reunião. A métrica estabeleceu três faixas: menos que 1/2 tomada por pessoa; 1/2 tomada por pessoa e; 1 tomada por pessoa. A referência utilizada para estabelecimento desta métrica foi estudo observacional em reuniões de coordenação de projetos. 
Mobiliário: O critério estabelecido para esta métrica diz respeito ao tamanho do apoio para equipamentos portáteis trazidos pelos participantes da reunião. A referência mínima foi baseada em uma carteira escolar com dimensões de $37 \mathrm{~cm}$ de largura por $33 \mathrm{~cm}$ de profundidade, referente a uma base mínima de apoio para um notebook. A métrica estabeleceu três faixas para a medida de largura e profundidade das áreas de trabalho: LxP < 37x33cm; 37x33cm < LxP < 80x61cm e; LxP > 81x62cm (PANERO; ZELNIK,1979).

Espaço Físico: Prever o mínimo de espaço de circulação ideal para locais de reunião. Os espaços de circulação devem ser adequados evitando interrupção das atividades da sala e possíveis acidentes durante a passagem, devido a fios, pés de mesa, suportes de equipamento. A métrica estabeleceu que distâncias mínimas de circulação a serem obedecidas devem ser classificadas em quatro faixas: $>1,00 \mathrm{~m}$; entre $1,00-0,80 \mathrm{~m}$; entre 0,80 $0,60 \mathrm{~m}$ e menor que $0,60 \mathrm{~m}$ (DIFFRIENT et al.,1979). Classificou-se cada uma das medidas obtidas de acordo com a faixa obtida na análise AHP e calculou-se a média desta classificação.

Conforto Ambiental: As questões relativas a conforto ambiental não irão alterar o leiaute de uma sala de reuniões. No entanto, devem ser consideradas como necessidades dos usuários e constaram como critério de análise. Temperatura confortável na sala de reuniões (entre $20^{\circ} \mathrm{C}$ e $23^{\circ} \mathrm{C}$ ) deve ser garantida (NR-17:1978), bem como o nível de ruído não deve ultrapassar a faixa entre 30-40 dB(A) (NBR10152:1987). A velocidade do ar não deve ser superior a $0,75 \mathrm{~m} / \mathrm{s}$ (NR-17:1978), a umidade relativa do ar não inferior a 40\% (NR-17:1978) e deve haver iluminação natural ou artificial adequada a uma sala de reunião em torno de 500 lux de luminância, 19 UGRL de limite de ofuscamento unificado e 80 Ra de índice de reprodução de cor mínimo (ABNT NBR ISO/CIE 8995-1:2013).

\subsection{Aplicação das métricas sobre os leiautes}

Foram selecionadas cinco métricas para serem aplicadas aos leiautes, sendo as 3 de visualização, a de interatividade e a de espaço relatadas no item 4.2 deste artigo. A justificativa para esta seleção diz respeito a serem variáveis dependentes do leiaute ao passo que infraestrutura, mobiliário e conforto ambiental são variáveis independentes deste fator. As métricas foram aplicadas aos 14 leiautes propostos. Primeiramente mediram-se os ângulos horizontais conforme o critério e métrica 1. Como exemplo, as notas obtidas com a medição para os participantes 1 a 6 foram indicadas em cores na Figura 2.

Figura 2 - Medição de ângulo horizontal
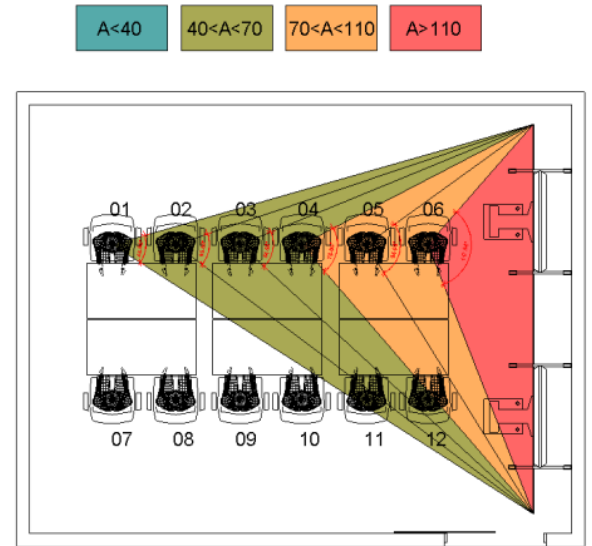

Fonte: Autores 
Em segundo lugar, mediu-se o índice de oclusão (métrica 2). Foram produzidas vistas 3D em software paramétrico (Revit) e utilizado software de pintura (Photoshop) para verificar a área de tela em oclusão, conforme a Figura 3.

Figura 3 - Índice de oclusão da tela

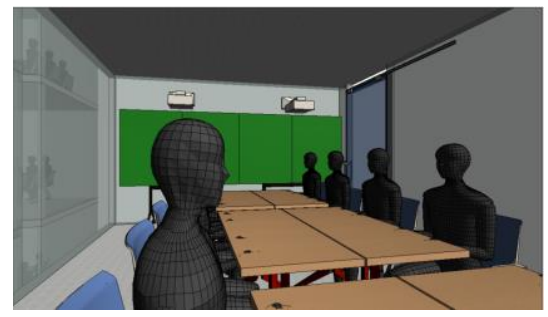

Fonte: Autores

Em terceiro lugar, mediu-se o Ângulo vertical (métrica 3), através da medição geométrica em corte, conforme a Figura 4.

Figura 4 - Ângulo Vertical

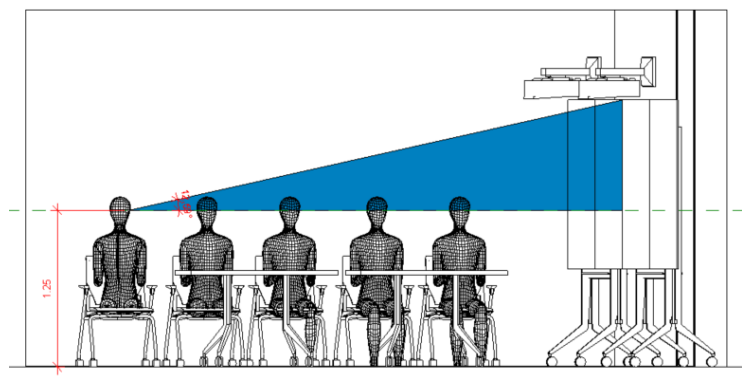

Fonte: Autores

Em quarto lugar foi feita a medição da Interatividade entre os usuários com a aplicação da métrica 4, com as medidas de distância e ângulo entre os participantes da reunião e a verificação de oclusão ou não. $E$, por fim, mediu-se a distância entre a perpendicular de cada assento à parede da sala atrás da cadeira (critério de Espaço físico).

\subsection{Análise multicritério com aplicação de AHP $^{2}$}

Com o propósito de entender qual a relação de importância entre todas as necessidades descritas no item 3 deste artigo, foi aplicado o método de decisão multicritério baseado na análise de comparações por pares (SAATY,1990). Utilizando a escala fundamental proposta pelo autor, foram colhidas as opiniões de 10 participantes de reuniões de coordenação de projeto. De acordo com estudo anterior (ADDOR; SANTOS, 2014) verificou-se que projetistas, coordenador e assistente de coordenador eram os papéis de maior frequência nas reuniões de coordenação de projetos. Os participantes que responderam às comparações foram: 4 coordenadores de projeto, 4 projetistas e 2 compatibilizadores de projeto. Todos tinham experiência com BIM e participam de reuniões de coordenação em BIM. Todos observaram imagens projetadas nas duas telas com conteúdos diferentes.

A importância relativa entre as os critérios visualização, interatividade, infraestrutura, mobiliário, espaço físico e conforto ambiental foram analisadas pelos 10 entrevistados, bem como os subcritérios movimentação do observador, porcentagem de oclusão da tela e

\footnotetext{
${ }^{2}$ Analytic Hierarchy Process
} 
ângulo para sua visualização, bem como distância, ângulo e obstrução de participante para interatividade.

\section{RESULTADOS}

Os resultados foram observados de acordo com a análise de comparação par a par e estão apresentados na tabela 1. Foram colhidas todas as comparações par a par, calculados os autovetores e autovalores segundo a metodologia AHP. Esta metodologia foi aplicada para categorizar alternativas em termos de classificação, intensidades ou graus de critérios (SAATY,1990). Estas graduações neste estudo são as notas relativas aos sub-subcritérios apresentadas no item 4.2 deste artigo. Após estabelecer a escala de prioridades para os critérios e subcritérios através da comparação por pares, os graus de diferença entre cada subcritério também foram comparados aos pares. Cada leiaute foi avaliado dentro de cada subcritério e critério. Após a soma global ponderada dos graus de prioridades, chegou-se a classificação para as alternativas de leiaute.

Os critérios visualização, interatividade, infraestrutura, mobiliário, espaço e conforto ambiental foram comparados dois a dois por dez participantes de reuniões. Os fatores de maior influência sobre a qualidade do leiaute foram: visualização, com 37,5\% de influência sobre o leiaute, seguido pela interatividade com $27,3 \%$ de influência, conforme a Tabela 1.

A mesma comparação dois a dois foi realizada pelos participantes para os subcritérios visualização e interatividade. O principal parâmetro de influência sobre o leiaute na visualização é a porcentagem de oclusão da tela com 55,7\%. Para o subcritério interatividade o fator ângulo que o observador tem que girar para interagir com outro observador teve a maior prioridade (42,5\%).

Após o estabelecimento dos fatores de prioridade dos critérios e subcritérios, as alternativas de leiaute foram analisadas. Os resultados obtidos na análise dos leiautes foram tabulados e ponderados pelos vetores de prioridade encontrados pela análise multicritério. Os 3 leiautes que receberam as maiores prioridades foram o L14 com nota 1,539 seguidos dos leiautes L13 e L1 com notas 1,537 e 1,517 respectivamente e, em quarta posição, o leiaute L7 com nota de 1,514, conforme as Figuras 5 e 6.

Tabela 1 - Resumo das médias dos vetores de prioridades dos critérios e sub critérios

\begin{tabular}{|c|c|c|c|c|c|c|}
\hline Critério & $\begin{array}{c}\text { Visualização } \\
0,3745\end{array}$ & $\begin{array}{c}\text { Interatividade } \\
0,2726\end{array}$ & $\begin{array}{c}\text { Infraestrutura } \\
0,1318\end{array}$ & $\begin{array}{c}\text { Mobiliário } \\
0,0763\end{array}$ & $\begin{array}{l}\text { Espaço } \\
0,0709\end{array}$ & $\begin{array}{c}\text { Cof Amb } \\
0,0739\end{array}$ \\
\hline $\begin{array}{l}\text { Sub Critério } \\
\text { Moviment. }\end{array}$ & $\begin{array}{c}\text { Mov Observ } \\
\text { ângulo Horiz } \\
0,2513\end{array}$ & $\begin{array}{c}\text { \% Oclusão Tela } \\
0,5573\end{array}$ & $\begin{array}{c}\text { Ângulo Vertical } \\
0,1914\end{array}$ & & & \\
\hline $\begin{array}{c}\text { Sub- } \\
\text { SubCritério } \\
\text { Ang Horiz }\end{array}$ & $\begin{array}{c}0<\mathrm{A}<40 \\
0,5086\end{array}$ & $\begin{array}{c}40<\mathrm{A}<70 \\
0,2978\end{array}$ & $\begin{array}{c}70<\mathrm{A}<110 \\
0,1348\end{array}$ & $\begin{array}{l}A>110 \\
0,0587\end{array}$ & & \\
\hline $\begin{array}{c}\text { Sub- } \\
\text { SubCritério } \\
\text { Ocl. Tela }\end{array}$ & $\begin{array}{c}0-5 \% \\
0,5308\end{array}$ & $\begin{array}{c}5,1-10 \% \\
0,2722\end{array}$ & $\begin{array}{c}10,1-15 \% \\
0,1459\end{array}$ & $\begin{array}{l}>15 \% \\
0,0511\end{array}$ & & \\
\hline $\begin{array}{c}\text { Sub- } \\
\text { SubCritério } \\
\text { Ang. Vert. }\end{array}$ & $\begin{array}{c}0<\mathrm{A}<30^{\circ} \\
0,7015\end{array}$ & $\begin{array}{c}30<\mathrm{A}<50^{\circ} \\
0,2108\end{array}$ & $\begin{array}{l}A>50^{\circ} \\
0,0877\end{array}$ & & & \\
\hline $\begin{array}{c}\text { Sub Critério } \\
\text { Interat. }\end{array}$ & $\begin{array}{c}\text { Obstrução } \\
0,2681\end{array}$ & $\begin{array}{l}\text { Ângulo } \\
0,4257\end{array}$ & $\begin{array}{c}\text { Distância } \\
0,3062\end{array}$ & & & \\
\hline $\begin{array}{c}\text { Sub- } \\
\text { SubCritério } \\
\text { Angulo }\end{array}$ & $\begin{array}{c}0-45^{\circ} \\
0,4908\end{array}$ & $\begin{array}{l}46-90^{\circ} \\
0,3203\end{array}$ & $\begin{array}{l}91-135^{\circ} \\
0,1324\end{array}$ & $\begin{array}{c}136-180^{\circ} \\
0,0566\end{array}$ & & \\
\hline
\end{tabular}




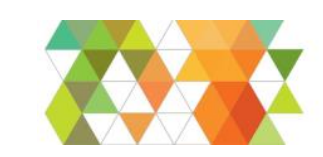

\section{TIC2015}

\begin{tabular}{|c|c|c|c|c|c|}
\hline $\begin{array}{c}\text { Sub- } \\
\text { SubCritério } \\
\text { Distância }\end{array}$ & $0-1,40 \mathrm{~m}$ & $1,41-2,40 \mathrm{~m}$ & $2,41-3,00 \mathrm{~m}$ & $>3,00 \mathrm{~m}$ & 0,0590 \\
\hline $\begin{array}{c}\text { Sub Critério } \\
\text { Espaço }\end{array}$ & 0,5043 & 0,2849 & 0,1517 & $\mathrm{D}<0,60 \mathrm{~m}$ & \\
& 0,4632 & $0,80<\mathrm{D}<1,00 \mathrm{~m}$ & $0,60<\mathrm{D}<0,80 \mathrm{~m}$ & 0,0537 & \\
\hline
\end{tabular}

Fonte: Autores

Figura 5 - Avaliação dos leiautes

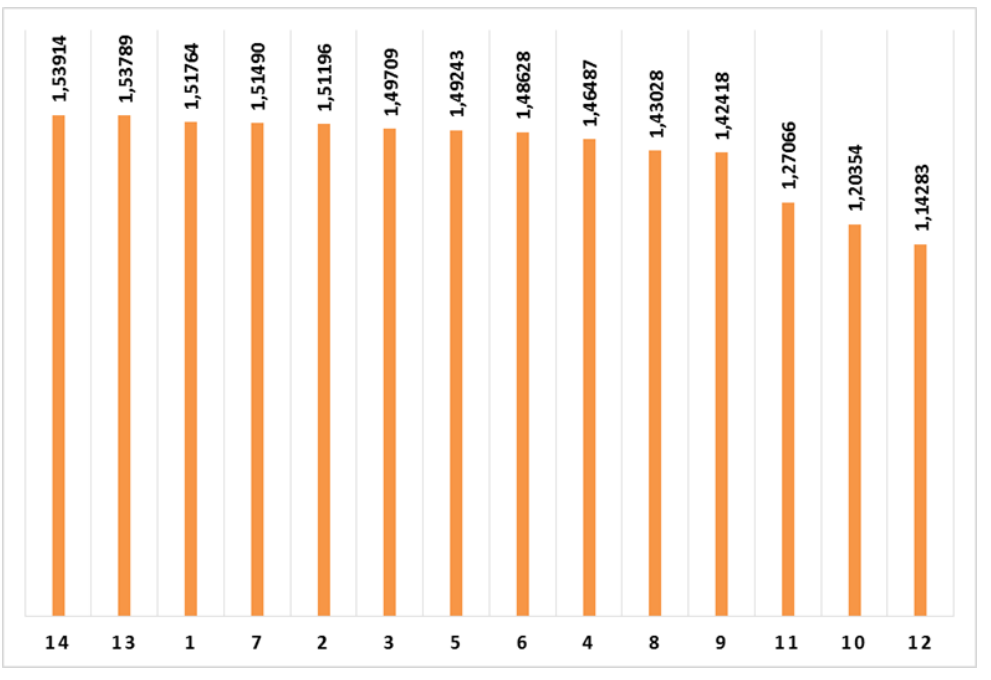

Fonte: Autores

Figura 6- Os quatro melhores leiautes segundo a análise

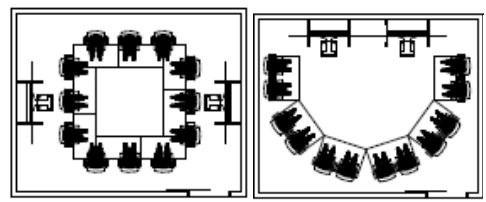

L14

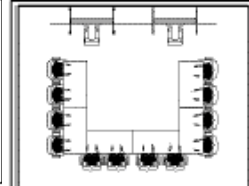

L1

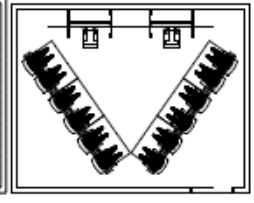

L7

Fonte: Autores

\section{CONCLUSÕES}

Neste artigo foi apresentada uma metodologia com critérios e métricas para avaliação da qualidade de leiautes em salas de coordenação BIM. A metodologia aplicada, AHP, é uma teoria descritiva de processo subjetivo intrínseco à investigação e necessita ser adaptada para a proposta de análise. Ao se tomar a opinião de pessoas na análise comparativa, podese ter uma subjetividade bastante grande. A inconsistência imposta pela escala de Saaty (1990) deve ser considerada dentro de 10\%. Desta forma, no caso estudado, devem-se reexaminar os 4 leiautes melhor avaliados, num refinamento da análise. Propõe-se para novas pesquisas o aprofundamento da análise sobre estes leiautes melhor avaliados. $O$ leiaute 14 obteve a melhor classificação apesar de ter recebido uma nota média baixa para visualização, uma vez que há pessoas de costas para ambas as telas. No entanto, sua nota de interatividade foi máxima, sendo que os três subcritérios que compõem a hierarquia de análise da interatividade foram bem atendidos e receberam notas bastante equilibradas, ou seja, tanto o ângulo, quanto a distância e a oclusão entre estes participantes estão bem 
classificados. O L13 obteve uma nota muito próxima do $L 14$, sendo que a nota de visualização do L13 foi a máxima obtida entre todos os leiautes, o que demonstra que os subcritérios de movimentação do observador, \% de oclusão da tela e máximo ângulo vertical estão muito bem atendidos. No entanto, a nota recebida para o critério interatividade distância foi a pior, o que demonstra que em termos de distância para comunicação, leiautes com formato muito aberto, tipo "U" não respondem bem a estes critérios. Por outro lado, o L13 obteve a melhor nota no critério interatividade - obstrução pois não há impedimento visual entre participantes neste leiaute, o que facilita muito a interação entre os participantes neste tipo de disposição. O L1, apesar de ficar na terceira colocação, obteve a segunda melhor nota de interatividade e, neste quesito, é melhor que o L13 pois existem mais pessoas de frente umas para as outras o que diminui a distância e o ângulo de interação. $O$ L7, apesar de quarto colocado, possui a segunda melhor nota em visualização da tela, sendo que há um equilíbrio entre a movimentação do observador, \% de oclusão da tela e máximo ângulo vertical, mostrando que, para uma boa visualização, leiautes em "V" também são uma boa opção. Outro fator a ser considerado em próximas pesquisas é a relação custo benefício entre as decisões. Apesar dos custos poderem ser incluídos nas decisões, devem ser desconsiderados até as alternativas serem avaliadas para que não aconteça de uma solução que ter ótimos benefícios mas ser inviável financeiramente. Deve-se analisar separadamente os custos de cada alternativa e depois julgá-las frente aos benefícios. $\mathrm{Na}$ pesquisa em questão, as salas receberam o mesmo número de equipamentos, espaço e pessoas e, por isso, a análise de custo não teria sentido. O interessante será analisar salas com diferentes equipamentos para se medir a relação custo benefício com equipamentos diferentes e custos diferentes.

Acredita-se que os dados gerados possam contribuir para novas investigações e proposições de instalações para melhor atender aos usuários de reuniões BIM.

\section{AGRADECIMENTOS}

Os autores agradecem a Pedro Henrique Pedrosa Torres por sua colaboração na coleta dos dados, à FINEP - Financiadora de Estudos e Projetos pelo apoio a esta pesquisa e ao CNPq - Conselho Nacional de Desenvolvimento Científico e Tecnológico e à Fundação de Amparo à Pesquisa do Estado de São Paulo (FAPESP) pelo apoio ao segundo autor.

\section{REFERÊNCIAS}

ASSOCIAÇÃO BRASILEIRA DE NORMAS TÉCNICAS. NBR ISO 9241-210:2011: Ergonomia da Interação Humano-Sistema.Parte 210: Projeto Centrado no Ser Humano para sistemas interativos. Rio de Janeiro, 2011.

. NBR ISO 9241-11 Requisitos Ergonômicos para o Trabalho com dispositivos de interação Visual Parte 11: Orientações Sobre Usabilidade. Rio de Janeiro, 2011.

1987.

NBR 10152:1987: Níveis de Ruído para Conforto Acústico-Procedimento. Rio de Janeiro, de Janeiro, 1987.

NBR ISSO/CIE 8995-1:2013: Iluminação de Ambientes de Trabalho - Parte 1: Interior. Rio

ADDOR, M.R.A.; SANTOS,E.T. Infraestrutura para uma Sala de Coordenação de Projetos em BIM: Avaliação dos Padrões de Comunicação e Requisitos. IN: XV ENCONTRO NACIONAL DE TECNOLOGIA DO AMBIENTE CONSTRUÍDO. Proceedings of...Universidade Federal de Alagoas, 2014. Disponível em: <http://www.infohab.org.br/entac2014/artigos/paper 615.pdf>. Acesso em : 10 dez. 2014. 
DENNIS, R. S. et al. 3D Model-Based Collaboration In Design Development and Construction of Complex Shaped Buildings. ITcon, v.13, p.258-285, junho 2008.

DIFFRIENT, N.; TILLEY, A.; BARDAGJY, J. Humanscale 1/2/3: a portfolio of information. New York. The MIT Press, 1979, 32p.

FRUCHTER, R. The Fishbowl: Degrees of Engagement in Global Teamwork. In: Smith, I. F. C. (ed.) Intelligent Computing In Engineering and Architecture. v. 4200, p.241-257,2006.

GOLPARVAR-FARD, M.; STAUB-FRENCH, S.; PO, B.; TORY, M. Requirements of a mobile interactive workspace for design development and coordination. JOINT INTERNATIONAL CONFERENCE ON COMPUTING AND DECISION MAKING IN CIVIL \& BUILDING ENGINEERING. 11. Proceedings of... Montreal: ASCE, 2006. p.3587-3596. Disponível em: <http://manigolparvar.com/conferencepapers/golparvarfardetal_2006_ASCE_ICCEBEXI.pdf>. Acesso em: 21 out. 2012.

INTERNATIONAL ORGANIZATION FOR STANDARDIZATION. ISO 9241-2:1992: Ergonomic Requirements for office work with visual display terminals (VDTs) Part 2: Guidance on Task Requirements., 1992.

JOHANSON, B.; FOX, A.; WINOGRAD, T. The Interactive Workspaces Project: Experiences with Ubiquitous Computing Rooms. IEEE Pervasive Computing,.v.1, n.2, p.67-74, abril 2002.

KASANEN, E.; LUKKA, K.; SIITONEN, A. The Constructive Approach in Management Accounting Research. Journal of Management Accounting Research, .v.5, p.243-264,1993.

LAHTI, H.; SEITAMAA-HAKKARAINEN, P.; HAKKARAINEN, K. Collaboration patterns in computer supported collaborative designing. Design Studies, v.25, n.4, p.351-371, julho 2004.

LEICHT, R. M. A framework for planning effective collaboration using interactive workspaces. 2009. Ph.D. Thesis (Architectural Engineering), Pennsylvania State University, 2009. 260p.

LISTON, K.; FISHER, M.; KUNZ, J. Requirements and Benefits of Interactive Information Workspaces in Construction. INTERNATIONAL CONFERENCE AND COMPUTING IN CIVIL AND BUILDING ENGINEERING, 8., Proceedings of..., Stanford University, 2000. Disponível em: <http://www.stanford.edu/group/4D/workspace/papers/ASCE2000-RequirementsKL.pdf>. Acesso em: 12 out. 2012.

McGRAW-HILL CONSTRUCTION The Business Value of BIM for Construction in Major Global Markets: How Contractors Around the World Are Driving Innovation With Building Information Modeling. SmartMarket Report. 2014.

MELHADO, S.B. et al. Coordenação de projetos de edificações. São Paulo. O Nome da Rosa, 2005. 120p.

MTE - Ministério do Trabalho e Emprego. NR-17-ERGONOMIA. Portaria GM n.3214, 1978. Disponível em: <http://portal.mte.gov.br/data/files/FF8080812BE914E6012BEFBAD7064803/ nr_17.pdf>. Acesso em 12 mar 2015.

OYEGOKE, A. The constructive research approach in project management research. International Journal of Managing Projects in Business, v.4, n4, p.573-595, 2011.

PANERO, J. ZELNIK, M. Human Dimension \& Interior Space - A Source Book of Design reference standards. New York: Watson-Guptill Publications,1979, 320p.

SAATY, T. L. How to make a decision: The Analytic Hierarchy Process. European Journal of Operational Research, v.48,n.1,p.9-26, Setembro 1990.

SANTOS, E. T. BIM Building Information Modeling: um salto para a modernidade na Tecnologia da Informação aplicada à Construção Civil. In: PRATINI, E.F.;SILVA JUNIOR, E.A. (eds.) Criação, representação e visualização digitais: tecnologias digitais de criação, representação e visualização no processo de projeto. 1ed. Brasília: Faculdade de Tecnologia da Universidade de Brasília, 2012, p. 25-62. 